\title{
Plasma response to $\mathrm{m} / \mathrm{n}=3 / 1$ resonant magnetic perturbation on J-TEXT Tokamak
}

\author{
Qiming $\mathrm{Hu}^{1}$, Jianchao $\mathrm{Li}^{1}$, Nengchao Wang ${ }^{1}$, Q. Yu ${ }^{2,3}$, Jie Chen ${ }^{1}$, Zhifeng Cheng ${ }^{1}$, \\ Zhipeng Chen ${ }^{1}$,Yonghua Ding ${ }^{1}$, Hai $\operatorname{Jin}^{1}$, Da Li ${ }^{1}$, Mao Li ${ }^{1}$, Yang Liu ${ }^{1}$, Bo Rao ${ }^{1}$,Lizhi Zhu ${ }^{1}$, Ge \\ Zhuang $^{1}$ and the J-TEXT Team \\ ${ }^{1}$ State Key Laboratory of Advanced Electromagnetic Engineering and Technology, School of \\ Electric and Electronic Engineering, HuazhongUniversity of Science and Technology, 430074, \\ Wuhan, China \\ ${ }^{2}$ Max-Planck-Institut für Plasmaphysik, 85748 Garching, Germany
}

Email: qiy@ipp.mpg.de

\begin{abstract}
The influence of resonant magnetic perturbations (RMPs) with a large $m / n=3 / 1$ component on electron density has been studied on J-TEXT tokamak by using externally applied static and rotating RMPs, where $m$ and $n$ are the poloidal and toroidal mode number, respectively. The detailed time evolution of electron density profile, measured by the polarimeter-interferometer, shows that the electron density $n_{\mathrm{e}}$ first increases (decreases) inside (around/outside) of the 3/1 rational surface (RS), and it is increased globally later together with enhanced edge recycling. Associated with field penetration, the toroidal rotation around the 3/1 RS is accelerated in co- $\mathrm{I}_{\mathrm{p}}$ direction and the poloidal rotation is changed from electron to ion diamagnetic drift direction. Spontaneous unlocking-penetration circles occur after field penetration if the RMPs amplitude is not strong enough. While for sufficiently strong RMPs, the $2 / 1$ locked mode is also triggered due to mode coupling, and the global density is increased. The field penetration threshold is found to be linearly proportional to the $n_{\mathrm{e}} \mathrm{L}$ (line-integrated density) at the $3 / 1$ RS but to $\left(n_{\mathrm{e}} \mathrm{L}\right)^{0.73}$ for the $n_{\mathrm{e}}$ at plasma core. In addition, for rotating RMPs with a large 3/1 component, field penetration causes global increase in electron density.
\end{abstract}

\section{Introduction}

Resonant magnetic perturbations (RMPs) generated by external saddle coils have important applications in present tokamak plasmas. RMPs have been demonstrated firstly on DIII-D to be a promising technique in mitigating/suppressing edge localized modes (ELMs) [1, 2], and subsequently ELMs control by RMPs were also investigated on JET [3], ASDEX-Upgrade [4], KATAR [5], MAST [6] and other tokamaks. RMPs have also been used to detect and correct the non-axisymmetric error field arising from misshapen or misplaced coils and their leads [7, 8], and many corresponding studies have been carried out on DIII-D [9-11], MAST [12] and KSTAR [13]. In addition, RMPs have also been used in active stabilization of resistive wall modes (RWMs) [14-16].

It is well known phenomena that the application of RMPs to mitigate ELMs or correct error field often leads to the change in the electron density, the so-called density pump-out [1-3, 17]. This change affects the L-H transition power [18-20] and fusion gain in the future burning plasmas. For the extrapolation of the application of RMPs to future devices with minimum degradation on particle and thermal confinement as well as minimum effects on L-H transition and MHD instabilities, it is important to understand the effects of RMPs on particle transport in both the Land H-mode plasmas.

The particle transport in the presence of RMPs has been extensively studied before. In L-mode plasmas, the density pump-out caused by applied RMPs is found to behave as a resonant feature, as observed on TEXTOR [21, 22] and LHD [23]. Depending on the RMPs spectrum as well as the

${ }^{3}$ Authors to whom any correspondence should be addressed. 
plasma parameters, either an improvement or a decrease in particle confinement is observed on Tore-Supra [24] and TEXTOR [22, 25, 26], and these experimental results can be reproduced by numerical modeling [27, 28]. On MAST the density pump-out is exhibited only when the RMP coil current is above a threshold, and the radial electric field $E_{\mathrm{r}}$ is also changed in a layer just inside the separatrix [29]. Comparisons on the change in the plasma density and rotation reveal that $E_{\mathrm{r}}$ is changed due to the modification of the toroidal/poloidal rotation, which might affects the particle transport [25, 29]. In addition, RMPs are found to cause the particle pinch to reverse from traditionally inward to outward, and the density at the plasma edge increases, while the density in the plasma core is reduced as observed on DIII-D [20]. For H-mode plasmas, it is observed that the density pump-out depends on the collisionality [30]. Studies carried out on DIII-D show that both the toroidal rotation and $E_{\mathrm{r}}$ are modified when density pump-out occurs [20], and the outward diffusion increases with application of RMPs whereas the inward pinch decreases [31]. To understand the physics basis of this topic, further studies on different devices are necessary.

Previous studies on J-TEXT exhibit resonant feature for the influence of RMPs on particle transport, and density pump-out occurs when $m / n=2 / 1$ locked mode is triggered by the 2/1 RMP [32], where $m$ and $n$ are the poloidal and toroidal mode number, respectively. Recent studies by using rotating 2/1 RMPs showed that RMPs improve (degrade) particle confinement when its frequency is higher (lower) than the natural 2/1 tearing mode (TM) frequency, and the amount of change in electron density is proportional to the difference between these two frequencies [33], being in good agreement with numerical predictions [27, 28].

In this paper, we focus on the plasma response to both static and rotating RMPs with its resonant position close to the plasma edge. In the experiment, the saddle coils is connected to generate a large 3/1 component, and the edge safety factor is chosen to be around 3.3. As a result, the field penetration of the 3/1 RMP is observed. The time evolution of the radial profile of electron density is measured by the multi-channel polarimeter-interferometer (POLARIS). For static RMPs, after field penetration the density is increased (decreased) inside (around/outside) the 3/1 RS firstly, and the global density is increased later. The toroidal rotation around the $3 / 1 \mathrm{RS}$ is accelerated in co- $I_{\mathrm{p}}$ direction and the poloidal rotation is changed from electron to ion diamagnetic drift direction. The change in electron density causes spontaneous unlocking-penetration circles, and the $2 / 1$ locked mode is also excited when the RMPs are strong enough. In addition, the scaling of penetration threshold on electron density is also studied. For rotating RMPs, field penetration causes an increase in global density. In Section 2 the experimental results are given: the experimental setup is described in section 2.1; the influence of static RMPs on density and the associated change in rotation are then presented in sections 2.2 and 2.3; in section 2.4 the results obtained by rotating RMPs are presented. Finally, discussions and summary are drawn in Section 3.

\section{Experimental setup and results}

\subsection{Experimental setup}

J-TEXT is a circular, medium sized, limiter tokamak with a major radius $\left(R_{0}\right)$ of $1.05 \mathrm{~m}$ and a minor radius $(a)$ of $0.25-0.3 \mathrm{~m}$ [34]. Recently, J-TEXT had been equipped with 12 in-vessel saddle coils, which can generate either static or rotating RMPs with a maximum frequency up to $6 \mathrm{kHz}$ [35]. In the present experiments, the connection of coil system is set to produce RMPs with a large $m / n=3 / 1$ resonant component. The spectrum of static RMPs calculated in vacuum condition is shown in figure 1(a), which is dominated by the $m / n=1 / 1$ and $3 / 1$ components for edge safety factor $q_{a}>3$. The $3 / 1$ component at the plasma edge is $b_{r}{ }^{3,1}(r=a)=1.2 \mathrm{Gs} / \mathrm{kA}$. Rotating RMPs are generated when the coil system is supplied with AC current, and the field spectrum calculated for 
the frequency of $5.3 \mathrm{kHz}$ is shown in figure 1(b). The eddy current in the vacuum vessel decreases the field amplitude of $3 / 1$ component to about $0.29 \mathrm{Gs} / \mathrm{kA}$ at plasma edge for $f_{\mathrm{RMP}}=5.3 \mathrm{kHz}$.
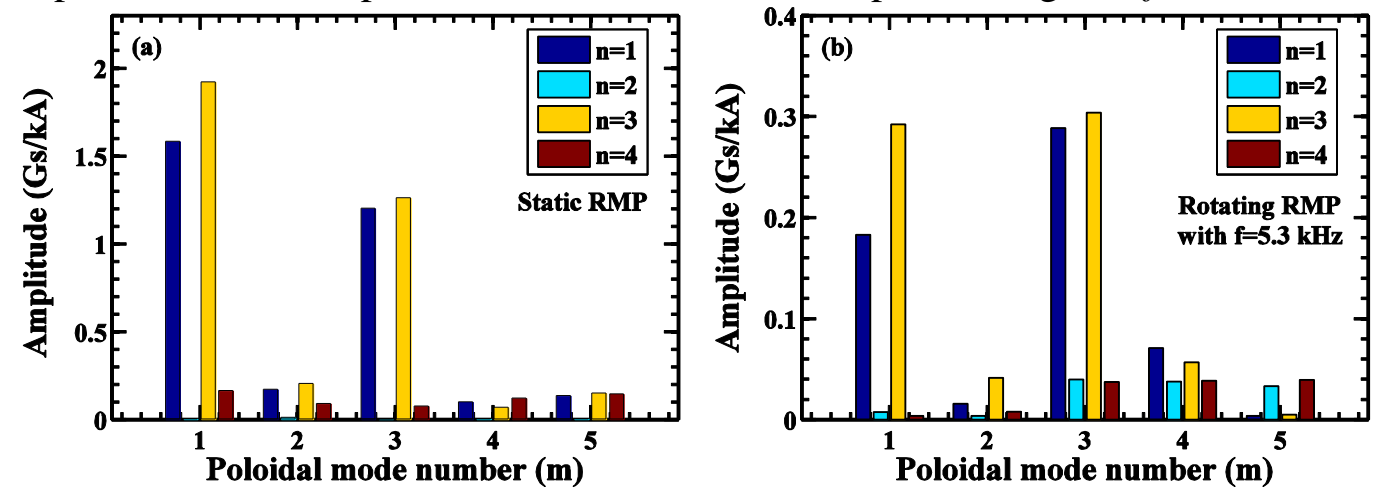

Figure 1.Spectrum of the radial magnetic field $b_{\mathrm{r}}$ generated by the saddle coils for (a) static RMPs and (b) rotating RMPs of a frequency $f_{\mathrm{RMP}}=5.3 \mathrm{kHz}$ at the plasma edge $(r=a)$ with poloidal mode number $(m)$ in the range of 1-5 and toroidal mode number $(n)$ in the range of 1-4. The magnetic field is calculated with vacuum condition, and the eddy current in the vacuum vessel is taken into account for calculating the spectrum of rotating RMPs with $f_{\mathrm{RMP}}=$ $5.3 \mathrm{kHz}$.

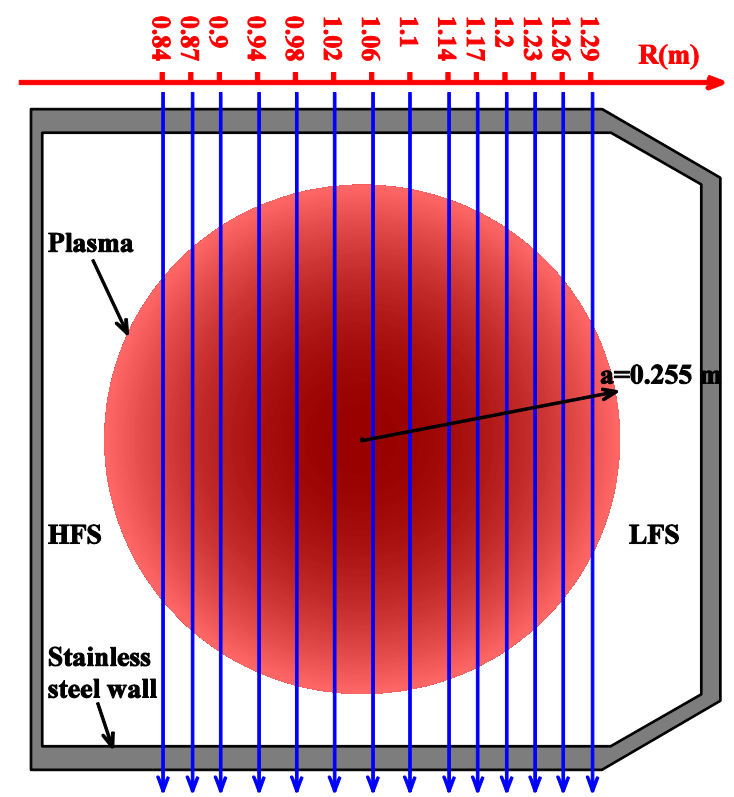

Figure 2. Diagram of multi-channel polarimeter-interferometer (POLARIS) for electron density measurement, which views the plasma vertically, and the radial positions are shown.

In the experiment, the electron density is measured by multi-channel polarimeter-interferometer (POLARIS) [36], which views the plasma vertically by 14 channels with the impact positions ranging from $R=0.84 \mathrm{~m}$ to $1.29 \mathrm{~m}$ as shown in figure 2, and the spatial temporal resolution are 30 $\mathrm{mm}$ and $1 \mu \mathrm{s}$. The electron density provided by the channel at/around $R=1.05 \mathrm{~m}$ is used to interpret the experimental results in the context. It will be noted in the text where the line-integrated density instead of line-averaged density is used. The MHD activity is measured by both the poloidal and toroidal probe arrays, where the poloidal array consists of 24 probes evenly distributed in poloidal direction. The locked mode detectors consist of two sets of saddle loops. Each set contains two loops installed at two opposite toroidal locations outside of the vacuum vessel [37]. The difference between the signals from two saddle loops in one set shows the perturbation with odd toroidal mode number $(n=1,3,5, \ldots)$. The electron temperature is measured by an 8 -channel heterodyne electron cyclotron emission (ECE) radiometer [38]. The toroidal and poloidal rotation of carbon V (CV) and carbon III (CIII) impurity at the edge of low field side are measured by a multi-channel high resolution spectrometer [39]. 


\subsection{Experimental results with static RMPs}

For the experimental results described in this subsection, the plasma parameters are as follows unless otherwise noted: major/minor radius $\left(R_{0} / a\right)$ is $1.05 \mathrm{~m} / 0.255 \mathrm{~m}$, plasma current $I_{\mathrm{p}}=160 \mathrm{kA}$, toroidal magnetic field $B_{\mathrm{t}}=1.65 \mathrm{~T}$, and the safety factor $q=3.3$ at the plasma edge. The core line-integrated electron density at $R=1.05 \mathrm{~m}$ is around $0.3-0.8 \times 10^{19} \mathrm{~m}^{-2}$. The central plasma rotation is observed to be in the electron diamagnetic drift direction, and the plasma is originally tearing stable.

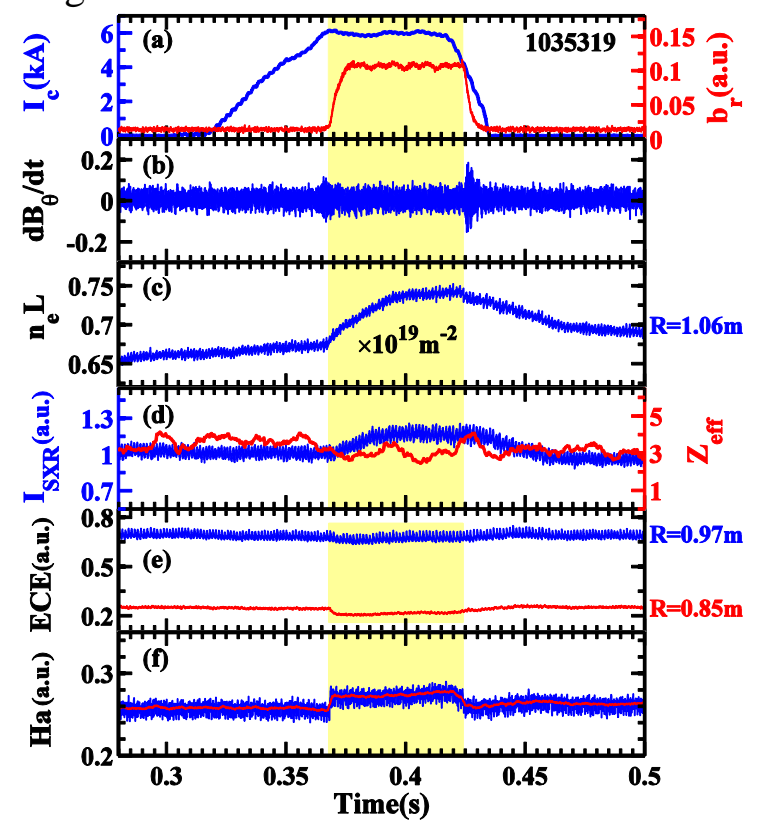

Figure 3. Shot 1035319, penetration of static 3/1 RMPs. Time evolution of (a) $I_{\mathrm{c}}$ and $b_{\mathrm{r}}$, (b) Mirnov signal $\mathrm{d} B_{\theta} / \mathrm{d} t$, (c) core integrated density $n_{\mathrm{e}} \mathrm{L}$, (d) core SXR radiation intensity $I_{\text {SXR }}$ and $Z_{\text {eff, }}$ (e) ECE signal at $R=0.97 \mathrm{~m}$ and $0.85 \mathrm{~m}$, and (f) edge H $\alpha$ signal.

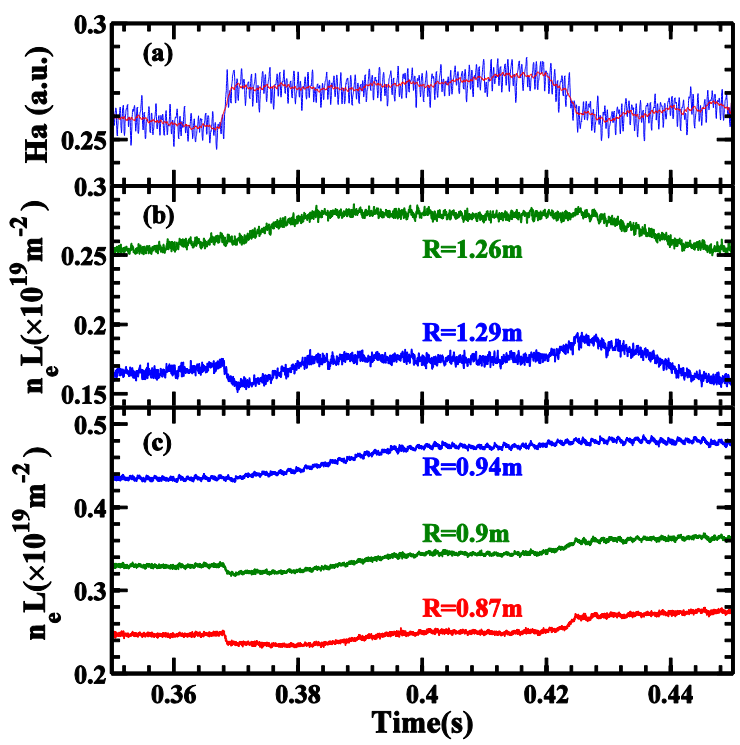

Figure 4.Corresponding to figure 3, time evolution of (a) Ha signal, integrated electron density at (b) LFS $(R$ $=1.26 \mathrm{~m}$ and $1.29 \mathrm{~m})$ and $(\mathrm{c}) \operatorname{HFS}(R=0.87 \mathrm{~m}, 0.9 \mathrm{~m}$, and $0.94 \mathrm{~m})$.

An example of the effect of static 3/1 RMPs on the electron density during field penetration is shown in figure 3 for shot 1035319. Before the application of RMPs, the plasma parameters nearly keep constant. The RMP coil current $\left(I_{\mathrm{c}}\right)$ is applied at $0.32 \mathrm{~s}$ (figure 3(a)), and it ramps up to the flattop of $6 \mathrm{kA}$ at $0.36 \mathrm{~s}$. Field penetration happens at $t=0.368 \mathrm{~s}$ as indicated by the fast growth of the locked mode detector signal $b_{\mathrm{r}}$ (red curve in figure 3(a)) when $I_{\mathrm{c}}$ ramps up to $5.9 \mathrm{kA}$. After field penetration, the line-integrated electron density $n_{\mathrm{e}} \mathrm{L}$ at $R=1.06 \mathrm{~m}$ begins to increase from $0.67 \times$ $10^{19} \mathrm{~m}^{-2}$ to $0.75 \times 10^{19} \mathrm{~m}^{-2}$ (figure 3(b)), with a relative change of $12 \%$. At the same time, the electron temperature decreases slightly close to plasma core $(R=0.97 \mathrm{~m})$ and more obvious at plasma edge $(R=0.85 \mathrm{~m})$ as shown in figure $3(\mathrm{~d})$. The soft X-ray emission (SXR) at plasma core increases and the effective $Z_{\text {eff }}$ is slightly decreased (figure 3(c)). The edge recycling increases slightly as indicated by the $\mathrm{H} \alpha$ signal at $\mathrm{r}=0.27 \mathrm{~m}$. At $t=0.425 \mathrm{~s}$ mode unlocking happens when $I_{\mathrm{c}}$ ramps down to $4 \mathrm{kA}$, and all the parameters begin to recover to their initial values. The unlocking threshold is only one third smaller than the penetration threshold, being smaller than that for the 2/1 and other modes as reported on COMPASS [40], J-TEXT [41, 42] and EXTRAP-T2R [43]. The possible reason for this is the change in plasma rotation, which will be discussed in section 2.3.

In figure 4, the time evolution of $n_{\mathrm{e}} \mathrm{L}$ around the 3/1 RS at both LFS and HFS are shown. For shot 1035319 the $3 / 1 \mathrm{RS}$ is found to be around $R=1.27 \mathrm{~m}$ at LFS and $0.9 \mathrm{~m}$ at HFS. It is found that $n_{\mathrm{e}} \mathrm{L}$ first decreases quickly around/outside of the 3/1 RS (for $R=1.29 \mathrm{~m}$ at LFS and $R \leq 0.9 \mathrm{~m}$ at HFS) right after field penetration. Afterwards the $n_{\mathrm{e}} \mathrm{L}$ increases both outside and inside the $3 / 1 \mathrm{RS}$. 

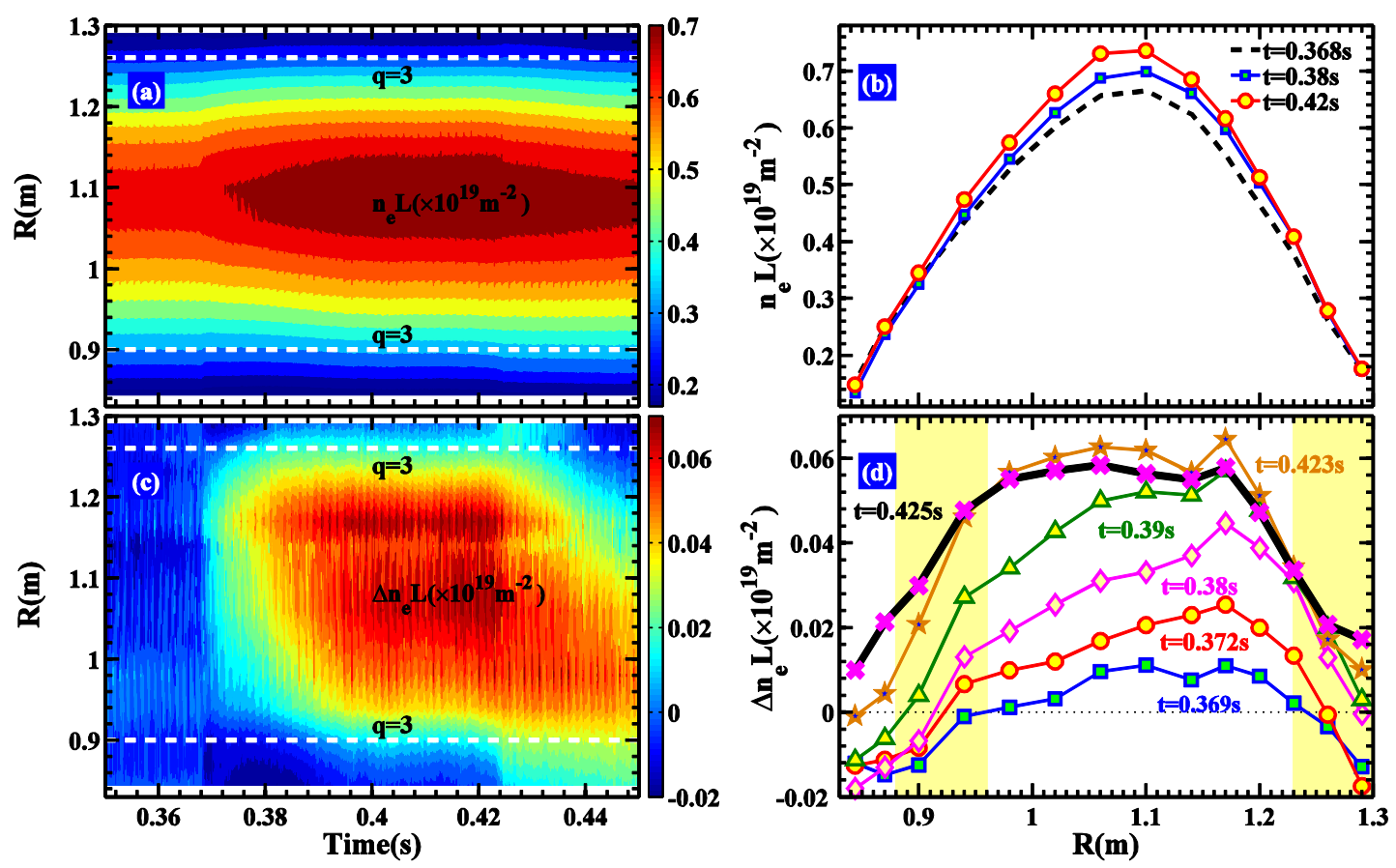

Figure 5.Corresponding to figure 3, time evolution of profile of $n_{\mathrm{e}} \mathrm{L}$ (a) in the interval of $0.35 \mathrm{~s}<t<0.45 \mathrm{~s}$ and (b) at $t=0.368 \mathrm{~s}, 0.38 \mathrm{~s}$, and $0.42 \mathrm{~s}$. Time evolution of profile of $\Delta n_{\mathrm{e}} \mathrm{L}=\left(n_{\mathrm{e}} \mathrm{L}-n_{\mathrm{e}} \mathrm{L}(t=0.368 \mathrm{~s})\right)$ (c) in the interval of $0.35 \mathrm{~s}<t<0.45 \mathrm{~s}$ and (d) at $t=0.369 \mathrm{~s}, 0.372 \mathrm{~s}, 0.38 \mathrm{~s}, 0.39 \mathrm{~s}, 0.423 \mathrm{~s}$, and $0.425 \mathrm{~s}$.

The time evolution of the profile of $n_{\mathrm{e}} \mathrm{L}$ and the changed density $\Delta n_{\mathrm{e}} \mathrm{L}=\left[\left(n_{\mathrm{e}} \mathrm{L}(t)-n_{\mathrm{e}} \mathrm{L}(t=\right.\right.$ $0.368 \mathrm{~s})$ ] are shown in figure 5(a)-5(d). During the field penetration, $n_{\mathrm{e}} \mathrm{L}$ first increases inside the $3 / 1$ RS but decreases around/outside of the 3/1 RS (figure 5(a) and 5(c)), and then the global density is raised as indicated by the profile of $n_{\mathrm{e}} \mathrm{L}$ at $t=0.38 \mathrm{~s}$ and $0.42 \mathrm{~s}$ before mode unlocking as shown in figure 5(b). The profiles of $\Delta n_{\mathrm{e}} \mathrm{L}$ at different times show that the gradient of density around the 3/1 RS is stronger (marked by the shadowed region in figure 5(d)), and it changes little during the raise of the profile. In addition, after mode unlocking, the profile of $\Delta n_{\mathrm{e}} \mathrm{L}$ at $t=0.425 \mathrm{~s}$ decreases little at plasma core but increases at plasma edge, and afterwards $\Delta n_{\mathrm{e}} \mathrm{L}$ declines with time. Results shown in figures 3 to 5 indicate that the penetration of the 3/1 RMP first increases (decreases) electron density inside (around/outside of) the 3/1 RS and then increases the global electron density. The electron temperature decreases slightly except a stronger reduction at plasma edge, and the edge recycling is slightly increased.

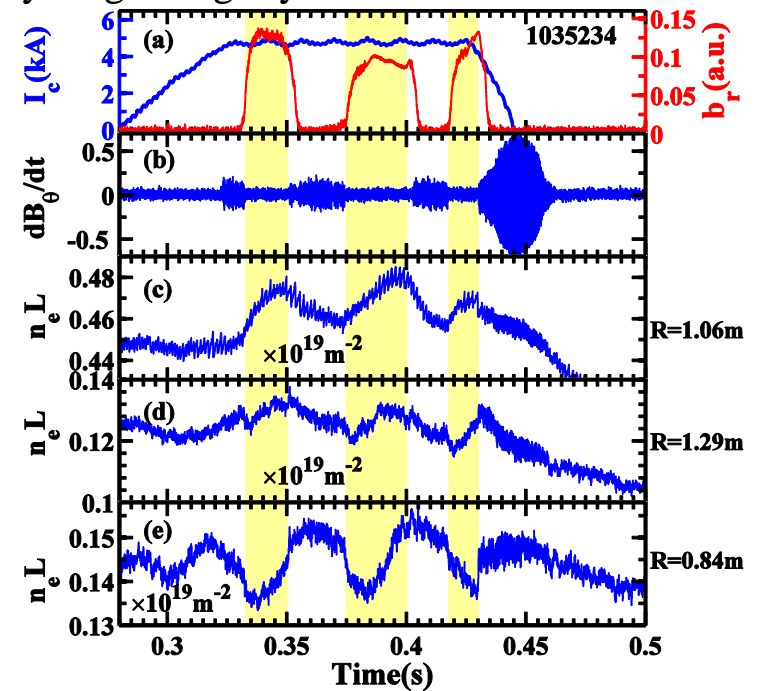

Figure 6. Shot 1035234, penetration of static 3/1 RMPs. Time evolution of $I_{\mathrm{c}}, b_{\mathrm{r}}, \mathrm{d} B_{\theta} / \mathrm{d} t$, integrated density $n_{\mathrm{e}} \mathrm{L}$ at $R=1.06 \mathrm{~m}, 1.29 \mathrm{~m}$ and $0.87 \mathrm{~m}$.

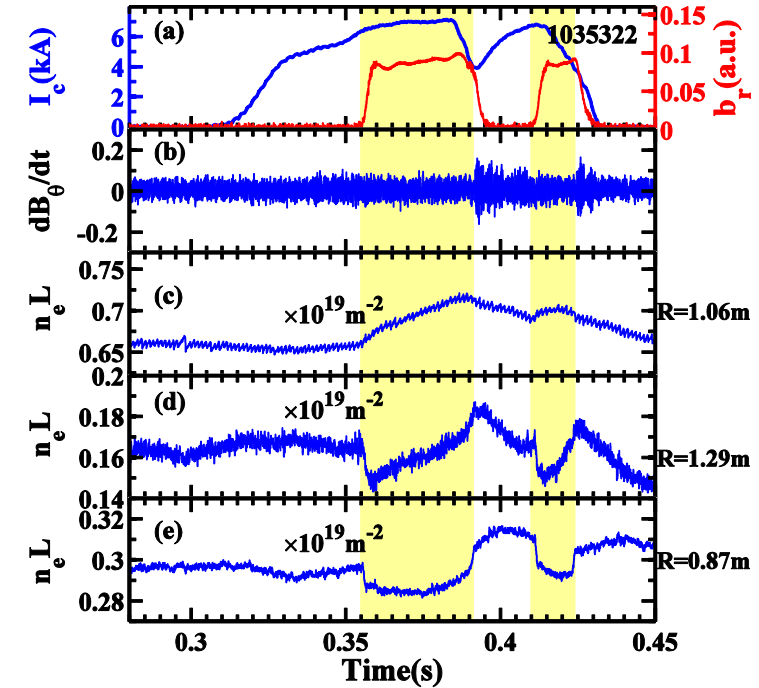

Figure 7. Shot 1035322, penetration of static $3 / 1$ RMPs. Time evolution of $I_{\mathrm{c}}, b_{\mathrm{r}}, \mathrm{d} B_{\theta} / \mathrm{d} t$, integrated density $n_{\mathrm{e}} \mathrm{L}$ at $R=1.06 \mathrm{~m}, 1.29 \mathrm{~m}$ and $0.87 \mathrm{~m}$.

After field penetration, the increased density may exceed the density threshold for penetration 
and then force the locked mode to unlock when the amplitude of RMPs is not strong enough. One typical result is shown in figure 6 for shot 1035234 , here $I_{\mathrm{c}}=4.7 \mathrm{kA}$ at the flattop and $n_{\mathrm{e}} \mathrm{L}$ is $0.45 \times 10^{19} \mathrm{~m}^{-2}$ before penetration. After field penetration at $t=0.33 \mathrm{~s}$, edge $n_{\mathrm{e}} \mathrm{L}$ decreases $($ at $R=$ $1.29 \mathrm{~m}$ and $0.84 \mathrm{~m}$ ) firstly, and later on it increases accompanying with the increase of central density $\left(n_{\mathrm{e}} \mathrm{L}\right.$ at $\left.R=1.06 \mathrm{~m}\right)$. At $t=0.35 \mathrm{~s}$, the central density increases to $n_{\mathrm{e}} \mathrm{L}=0.48 \times 10^{19} \mathrm{~m}^{-2}$ and the edge density exceeds the density threshold for penetration, so that mode unlocking happens spontaneously. The global density decreases after mode unlocking, which is similar to the result as shown in figure 5. When the density decreases to the value near the density threshold, field penetration happens again at $t=0.365 \mathrm{~s}$ and $0.42 \mathrm{~s}$, respectively. When first decreasing and then increasing $I_{\mathrm{c}}$ after field penetration, the penetration-unlocking circle is also observed as shown in figure 7 for shot 1035322 .

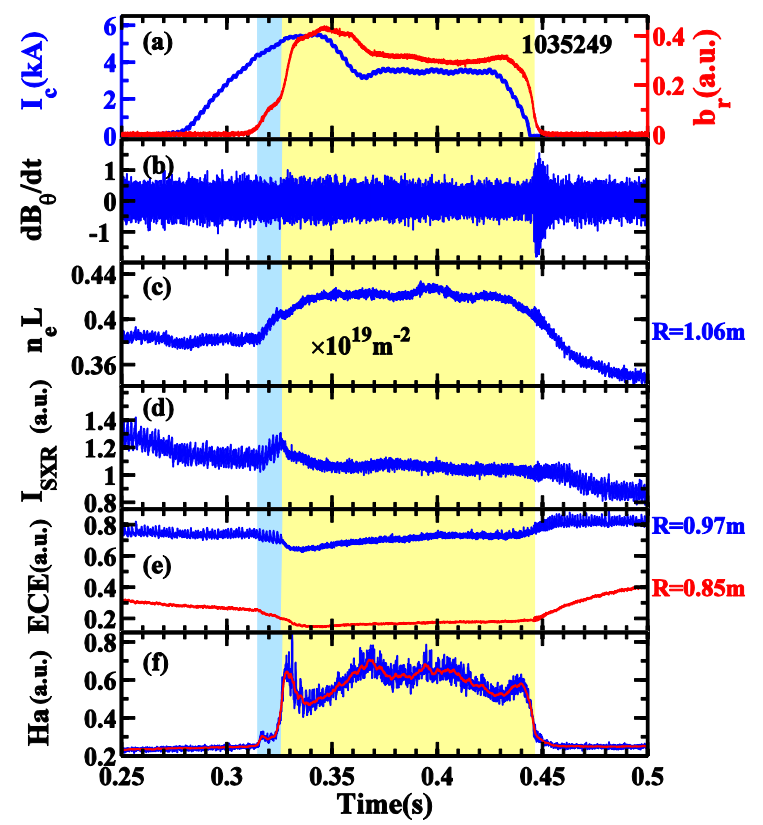

Figure 8. Shot 1035249, penetration of static 3/1 RMPs and excitation of 2/1 locked mode. Time evolution of $I_{\mathrm{c}}$, $b_{\mathrm{r}}, \mathrm{d} B_{\theta} / \mathrm{d} t, n_{\mathrm{e}} \mathrm{L}$ at plasma core, core $\mathrm{SXR}$ radiation intensity $I_{\mathrm{SXR}}$, ECE signal at $R=0.97 \mathrm{~m}$ and $0.85 \mathrm{~m}$, and edge $\mathrm{H} \alpha$ signal.

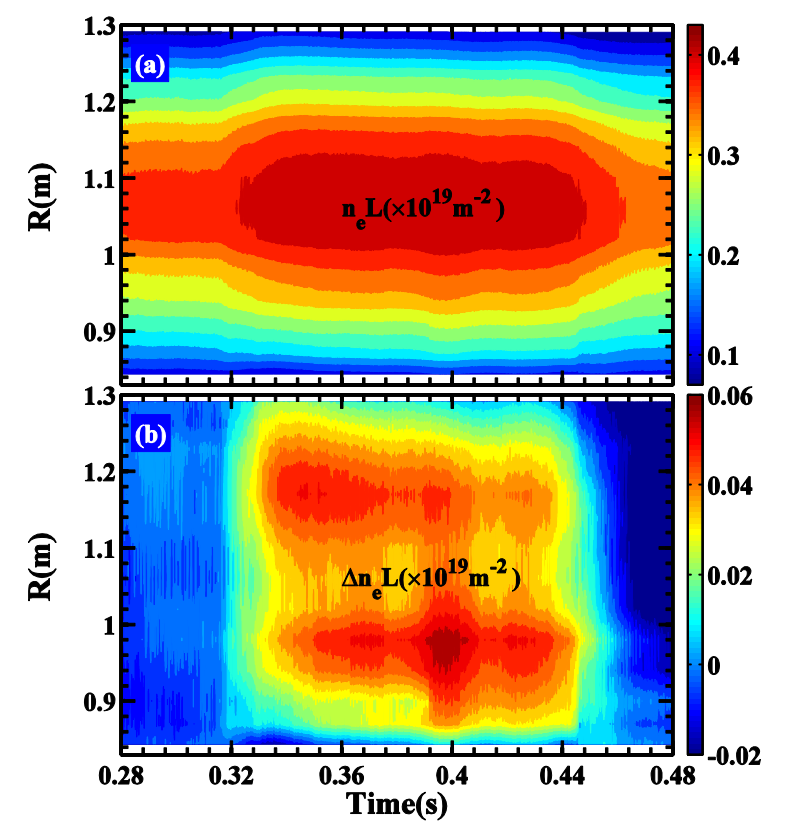

Figure 9. Corresponding to figure 8, time evolution of profile of (a) $n_{\mathrm{e}} \mathrm{L}$ and (b) $\Delta n_{\mathrm{e}} \mathrm{L}=\left(n_{\mathrm{e}} \mathrm{L}-n_{\mathrm{e}} \mathrm{L}(t=0.28 \mathrm{~s})\right)$ in the interval of $0.28 \mathrm{~s}<t<0.48 \mathrm{~s}$.

For the plasma with lower equilibrium density, the 3/1 locked mode together with a weak 2/1 component of RMPs may trigger an additional 2/1 locked mode when the applied RMPs are strong enough relative to penetration threshold, as shown in figure 8 for shot 1035249 . Here, $I_{\mathrm{c}}=5.5 \mathrm{kA}$ at the first flattop and $n_{\mathrm{e}} \mathrm{L}=0.37 \times 10^{19} \mathrm{~m}^{-2}$. During the time interval of $0.315 \mathrm{~s}<t<0.325 \mathrm{~s}$, a $3 / 1$ locked mode is triggered and the changes in all measured signals are similar to those shown in figure 3. However, for $0.325 \mathrm{~s}<t<0.445 \mathrm{~s}$, the $b_{\mathrm{r}}$ signal, the $n_{\mathrm{e}} \mathrm{L}$ at plasma core and the Ho signal further increase to a higher level (figure 8(a), (b), (e)), while the electron temperature at both plasma core and edge decrease substantially, and the SXR signal decreases slightly. By analyzing the Mirnov signal right after mode unlocking, it is found that both the $2 / 1$ and the $3 / 1$ modes exist, indicating that a $2 / 1$ locked mode is also triggered after the field penetration of 3/1 RMPs. Differing from the usual occurrence of $2 / 1$ locked mode with density pump-out $[32,44]$, in figure 8 the plasma density increases after the trigger of $2 / 1$ locked mode. Corresponding to figure 8 , the time evolution of the radial profile of $n_{\mathrm{e}} \mathrm{L}$ and $\Delta n_{\mathrm{e}} \mathrm{L}=\left(n_{\mathrm{e}} \mathrm{L}-n_{\mathrm{e}} \mathrm{L}(t=0.28 \mathrm{~s})\right)$ are shown in figure 9(a) and 9 (b), respectively. It is found that, the global density is increased except the slight reduction at the very edge of HFS during the interval of $0.325 \mathrm{~s}<t<0.35 \mathrm{~s}$. Besides, the profile of $\Delta n_{\mathrm{e}} \mathrm{L}$ peaks at $R$ $=1.18 \mathrm{~m}$ and $0.96 \mathrm{~m}$ of LFS and HFS, revealing that stronger density gradient is formed outside both the radius. Further experiments reveal that the phenomena shown in figures 8 and 9 are repeatable, and the increased density can be maintained with a lower $I_{\mathrm{c}}$ after field penetration as 
shown in figure $8(\mathrm{a})$, but disruption may happen later if $I_{\mathrm{c}}$ has not been lowered down from the flattop.

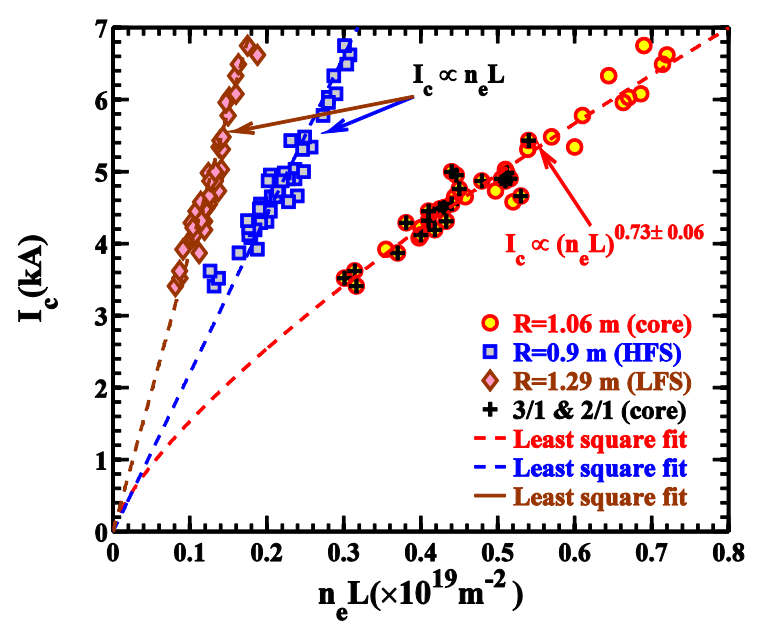

Figure 10. Data obtained from density scanning discharges with fixed plasma current $I_{\mathrm{p}}=160 \mathrm{kA}$, toroidal field $B_{\mathrm{t}}=1.65 \mathrm{~T}$ and $q_{\mathrm{a}}=3.3$, showing the line-integrated electron density $n_{\mathrm{e}} \mathrm{L}$ versus the amplitude of RMP coil current $I_{\mathrm{c}}$. The red circles (O) represent the penetration threshold of $n_{\mathrm{e}} \mathrm{L}$ at $R=1.06 \mathrm{~m}$, the blue squares $(\square)$ and brown diamonds $(\diamond)$ represent the penetration threshold of $n_{\mathrm{e}} \mathrm{L}$ at $R=0.87 \mathrm{~m}$ and $1.29 \mathrm{~m}$. The pluses $(+)$ represent the shots that $2 / 1$ locked mode is excited. The dashed curves are obtained from the least-squares fit for the penetration cases using a power law, and the obtained density scaling is $I_{\mathrm{c}} \propto\left(n_{\mathrm{e}} \mathrm{L}\right)^{0.73 \pm 0.06}$ at $R=1.06 \mathrm{~m}$ and $I_{\mathrm{c}} \propto n_{\mathrm{e}} \mathrm{L}$ at $R=$ $0.89 \mathrm{~m}$.

The relationship between the penetration threshold and the electron density is investigated by scanning the central $n_{\mathrm{e}} \mathrm{L}$ from $0.3 \times 10^{19} \mathrm{~m}^{-2}$ to $0.75 \times 10^{19} \mathrm{~m}^{-2}$. The RMPs coil current $I_{\mathrm{c}}$ at the moment of field penetration is taken to be the penetration threshold. The relationship between the penetration threshold and the $n_{\mathrm{e}} \mathrm{L}$ at different radius is shown in figure 10. The red circles (O) represent the penetration threshold for the $n_{\mathrm{e}} \mathrm{L}$ at $R=1.06 \mathrm{~m}$, and the blue squares $(\square)$ and brown diamonds $(\diamond)$ represent the penetration threshold for the $n_{\mathrm{e}} \mathrm{L}$ at $R=0.9 \mathrm{~m}$ and $1.29 \mathrm{~m}$. Here, the radial positions of $R=0.9 \mathrm{~m}$ and $1.29 \mathrm{~m}$ are the closest chords outside of the 3/1 RS on the HFS and LFS. The pluses (+) represent the shots that the $2 / 1$ locked mode is also excited after the field penetration of the 3/1 RMP. The corresponding electron density is measured at the moment of penetration. It is found that the penetration threshold increases with the density. The least-squares fit for the penetration data using a power law is marked by the dashed curves and displays a density scaling of $I_{\mathrm{c}} \propto\left(n_{\mathrm{e}} \mathrm{L}\right)^{0.73 \pm 0.06}$ for the central density at $R=1.06 \mathrm{~m}$, and $I_{\mathrm{c}} \propto n_{\mathrm{e}} \mathrm{L}$ for the edge density at $R=0.9 \mathrm{~m}$ and $1.29 \mathrm{~m}$ (around 3/1 RS). These results are different from the scaling of penetration threshold for $2 / 1$ mode on other tokamaks, which usually scale in linear for the central density [45-47]. Another scaling $I_{\mathrm{c}} \propto n_{\mathrm{e}}^{0.5}$ is also found on TEXTOR [48] and J-TEXT [49].

\subsection{Change in plasma rotation}

Previous studies on TEXTOR show that the change in electron density correlates with the change in plasma rotation and $E_{\mathrm{r}}[25]$, and numerical modeling also finds that, depending on the direction of plasma rotation, the density can be either increased or decreased [27, 28]. During the experiment with static RMPs, both the toroidal and poloidal rotation of CV and CIII have been measured, and CV and CIII cover the region of 0.75-0.85 $a$ and 0.85-0.95 $a$ (around the 3/1 RS at LFS), respectively. Corresponding to figure 6 , the time evolution of both the toroidal and poloidal rotation of $\mathrm{CV}$ and $\mathrm{CIII}$ are shown in figure 11 . Here, the positive value indicates the co- $I_{\mathrm{p}}$ direction or the ion diamagnetic drift direction for toroidal and poloidal rotation, respectively. It is found that $\mathrm{V}_{\phi}(\mathrm{CV})$ changes from 0 to nearly $4 \mathrm{~km} / \mathrm{s}$, while $\mathrm{V}_{\phi}(\mathrm{CIII})$ changes little. The poloidal rotation velocity $V_{\theta}(C V)$ increases slightly and $V_{\theta}(C I I I)$ increases from $-1.5 \mathrm{~km} / \mathrm{s}$ to $1 \mathrm{~km} / \mathrm{s}$ in ion diamagnetic drift direction $\left(f_{* \text { ion }}\right)$. After mode unlocking, all the rotation velocities recover to their initial values. 


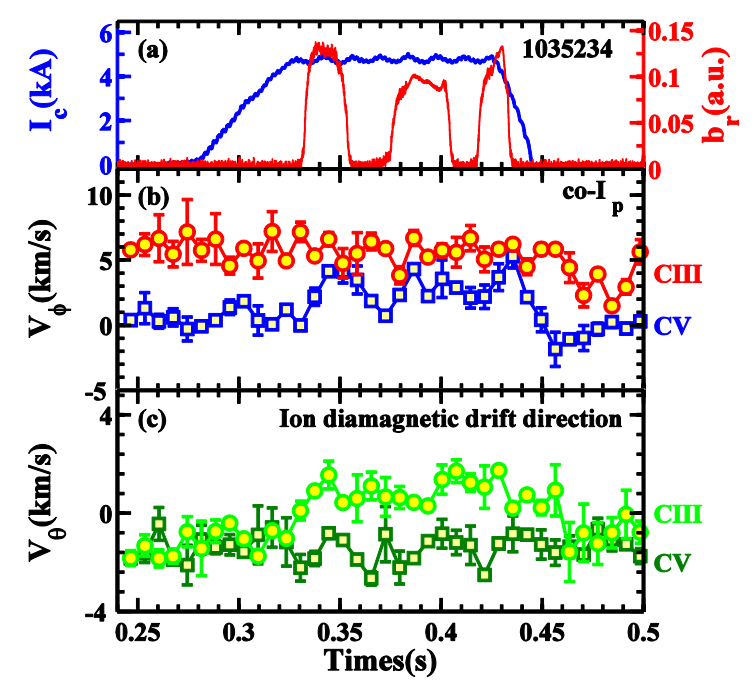

Figure 11. Corresponding to figure 6 , the time evolution of (a) $I_{\mathrm{c}}$ and $b_{\mathrm{r}}$, (b) toroidal rotation of $\mathrm{CV}$ (squares) and CIII (circles) and (c) poloidal rotation of CV (squares) and CIII (circles).

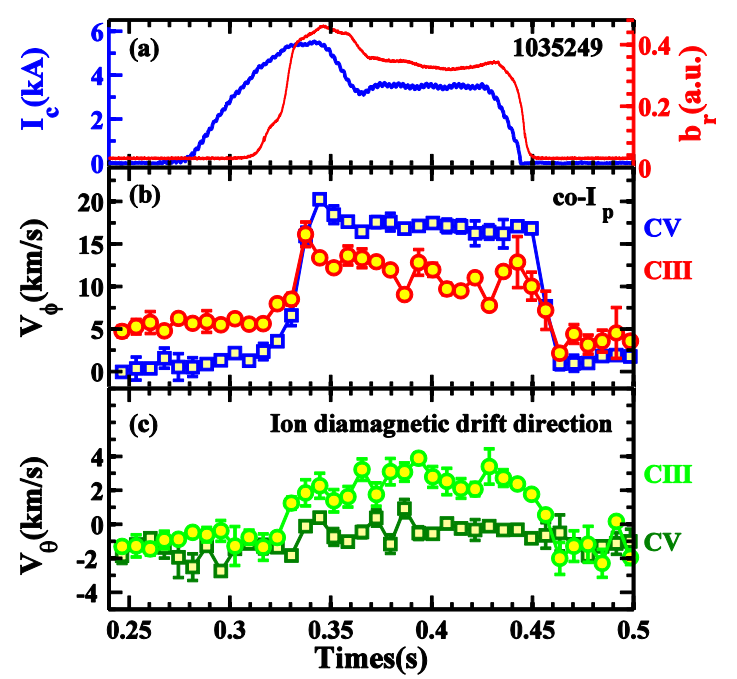

Figure 12. Corresponding to figure 8 , the time evolution of (a) $I_{\mathrm{c}}$ and $b_{\mathrm{r}}$, (b) toroidal rotation of CV (squares) and CIII (circles) and (c) poloidal rotation of $\mathrm{CV}$ (squares) and CIII (circles).

When the amplitude of RMPs is strong enough and a 2/1 locked mode is excited, the change in rotation is similar to that shown in figure 11. Corresponding to figure 8 , the time evolution of both the $\mathrm{V}_{\phi}$ and $\mathrm{V}_{\theta}$ of CV and CIII are shown in figure 12. It is found that $\mathrm{V}_{\phi}(\mathrm{CV})$ and $\mathrm{V}_{\phi}(\mathrm{CIII})$ are increased to $17.5 \mathrm{~km} / \mathrm{s}$ and $11.2 \mathrm{~km} / \mathrm{s}$ in the co- $I_{\mathrm{p}}$ direction, respectively, which is similar to the results of field penetration and mode locking observed on COMPASS [40] and J-TEXT [50]. For poloidal rotation, $\mathrm{V}_{\theta}(\mathrm{CV})$ and $\mathrm{V}_{\theta}(\mathrm{CIII})$ increases from $-2 \mathrm{~km} / \mathrm{s}$ to 0 and $-1.5 \mathrm{~km} / \mathrm{s}$ to $3 \mathrm{~km} / \mathrm{s}$ in co$f_{\text {*ion }}$ direction.
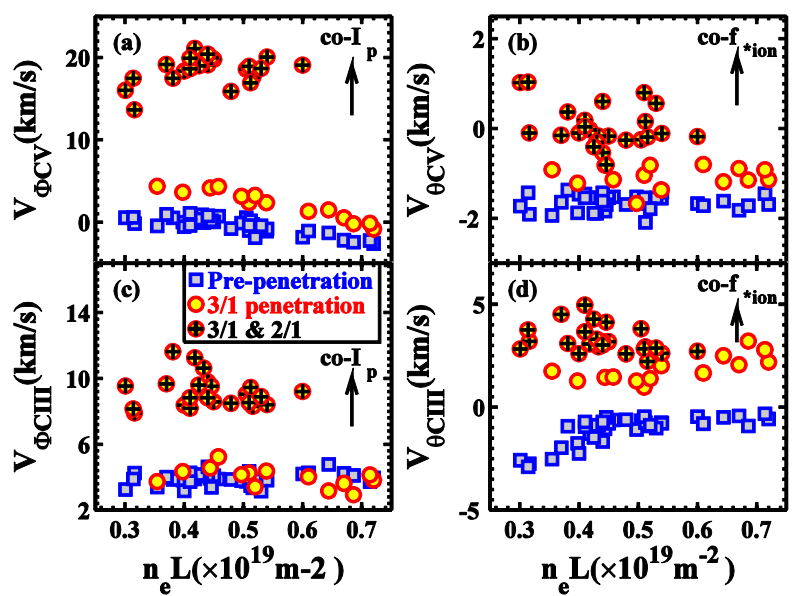

Figure 13. Corresponding to figure 10, the toroidal and poloidal rotation before (squares) and after (circles) field penetration are shown as a function of core line-integrated density $n_{e} L$. (a) $V_{\phi}$ and (b) $V_{\theta}$ of CV, (c) $V_{\phi}$ and (d) $V_{\theta}$ of CIII.

Corresponding to figure 10 , the toroidal and poloidal rotation before and after field penetration are shown as a function of core line-integrated density in figure 13. It is found that before the penetration of RMPs, $V_{\phi}$ and $V_{\theta}$ decrease slightly towards counter- $I_{p}$ and the electron diamagnetic drift direction respectively with increasing the electron density. The penetration of 3/1 RMPs causes a increase of $\sim 4 \mathrm{~km} / \mathrm{s}$ in the co- $I_{p}$ direction for $\mathrm{V}_{\phi}(\mathrm{CV})$, while $\mathrm{V}_{\phi}(\mathrm{CIII})$ changes little. $\mathrm{V}_{\theta}(\mathrm{CV})$ and $\mathrm{V}_{\theta}(\mathrm{CIII})$ increase by about $1 \mathrm{~km} / \mathrm{s}$ and $3 \mathrm{~km} / \mathrm{s}$ towards co- $I_{\mathrm{p}}$ and ion diamagnetic drift direction. When a $2 / 1$ locked mode is further excited, $\mathrm{V}_{\phi}(\mathrm{CV})$ and $\mathrm{V}_{\phi}(\mathrm{CIII})$ are substantially accelerated in the co- $I_{\mathrm{p}}$ direction, while $\mathrm{V}_{\theta}(\mathrm{CV})$ and $\mathrm{V}_{\theta}(\mathrm{CIII})$ are accelerated in ion diamagnetic drift direction. By comparing to the results in section 2.2 and in figure 12, a correlation between the change in density and rotation is found: associated with the increased electron density, the poloidal rotation around the $3 / 1 \mathrm{RS}$ is accelerated to co- $f *_{\text {ion }}$ direction, while the rotation inside the $3 / 1 \mathrm{RS}$ changes little. And 
this correlation is consistent with the numerical prediction [27, 28] and experimental observations [33]. In addition, the plasma rotation around 3/1 RS is low and the change in rotation is moderate after field penetration, resulting in that the mode unlocking occurs easily when ramping down RMP current, as shown in figures 3 and 6.

\subsection{Experimental results with rotating RMPs}

Similar to the results presented in section 2.2, rotating RMP is also applied to study the change in electron density and it rotates in the counter- $I_{\mathrm{p}}$ (electron diamagnetic drift) direction. In the experiments, the plasma parameters are as follows: major/minor radius $\left(R_{0} / a\right)$ is $1.05 \mathrm{~m} / 0.255 \mathrm{~m}$, plasma current $I_{\mathrm{p}}=160 \mathrm{kA}$, toroidal magnetic field $B_{\mathrm{t}}=1.55 \mathrm{~T}$, and the safety factor $q_{\mathrm{a}}=3.14$ at the plasma edge. The core line-integrated electron density at $R=1.05 \mathrm{~m}$ is around $0.4-0.5 \times 10^{19} \mathrm{~m}^{-2}$.

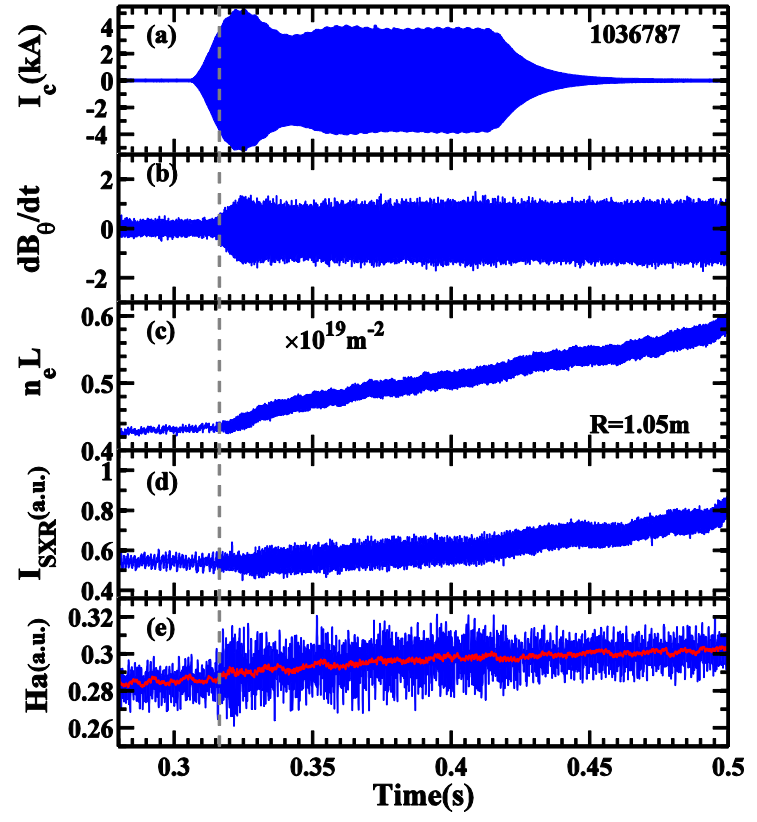

Figure 14. Shot 1036787 , field penetration of rotating $3 / 1$ RMPs with $f_{\mathrm{RMP}}=5.3 \mathrm{kHz}$. Time evolution of $I_{\mathrm{c}}$, $\mathrm{d} B_{\theta} / \mathrm{d} t, n_{\mathrm{e}} \mathrm{L}$ at $R=1.05 \mathrm{~m}$, core $\mathrm{SXR}$ radiation intensity $I_{\mathrm{SXR}}$, and edge $\mathrm{H} \alpha$ signal.

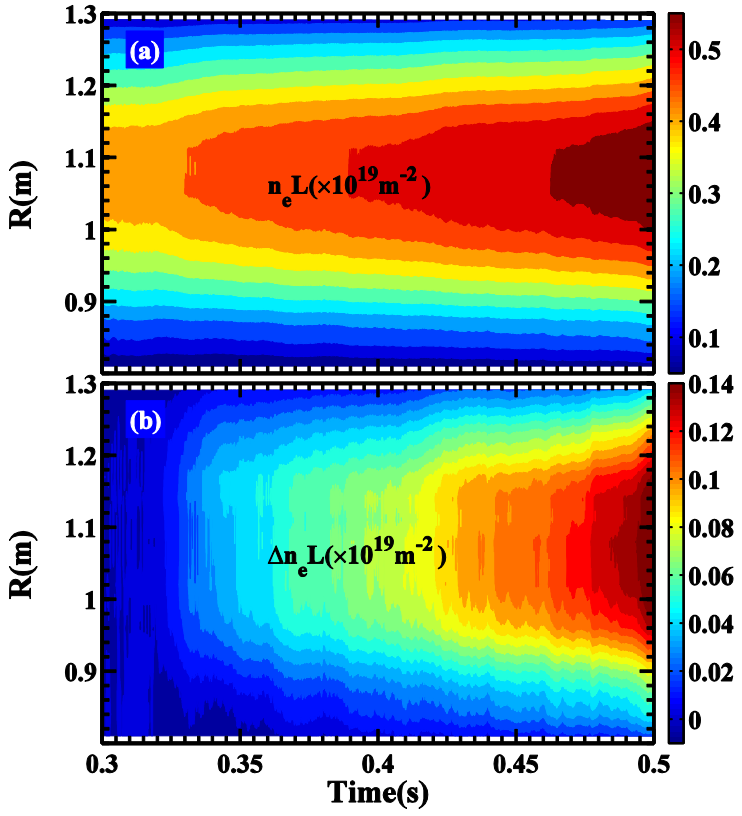

Figure 15. Corresponding to figure 14, time evolution of the radial profile of (a) $n_{\mathrm{e}} \mathrm{L}$ and (b) the changed density $\Delta n_{\mathrm{e}} \mathrm{L}=\left(n_{\mathrm{e}} \mathrm{L}-n_{\mathrm{e}} \mathrm{L}(t=0.32 \mathrm{~s})\right)$ in the interval of $0.3 \mathrm{~s}<t<0.5 \mathrm{~s}$.

An example of the effect of rotating RMPs on electron density is shown in figure 14 with the rotating frequency of $f_{\mathrm{RMP}}=5.3 \mathrm{kHz}$ and $I_{\mathrm{c}}=4 \mathrm{kA}$ at the flattop. Before applying RMPs, the plasma is tearing stable and the parameters keep constant. At $t=0.305 \mathrm{~s}$, rotating RMPs are turned on, field penetration happens later at $\mathrm{t}=0.315 \mathrm{~s}$ when $I_{\mathrm{c}}$ ramps up to $3.1 \mathrm{kA}$ (figure $14(\mathrm{~b})$ ), so that a rotating $3 / 1 \mathrm{TM}$ is triggered and driven to rotate synchronously with RMPs at $5.3 \mathrm{kHz}$. And this $3 / 1$ rotating TM does not vanish after turning off RMPs at $t=0.42 \mathrm{~s}$, having a frequency $4 \mathrm{kHz}$. After field penetration, the line-integrated electron density $n_{\mathrm{e}} \mathrm{L}$ at $R=1.05$ m increases from 0.43 to $0.6 \times 10^{19}$ $\mathrm{m}^{-2}$, with a relative change of $40 \%$. The density fluctuation correlated with rotating $3 / 1 \mathrm{TM}$ is observed (figure 14 (c)). The time evolution of the radial profile of $n_{\mathrm{e}} \mathrm{L}$ and the relative (to that at $t=$ 0.32 ) density change $\Delta n_{\mathrm{e}} \mathrm{L}$ as shown in figure 15(a) and 15(b) indicates that the global density is increased, and no opposite change in density is observed around the 3/1 RS. The SXR signal increases continuously associated with the increase of density (figure 14(d)), and the edge recycling increases slightly as indicated by the $\mathrm{H} \alpha$ signal at $r=0.27 \mathrm{~m}$ (figure 14(e)). Results shown in figures 14 and 15 indicate that the field penetration of 3/1 rotating RMPs increases the electron density in this case, and such an increase happens globally. 


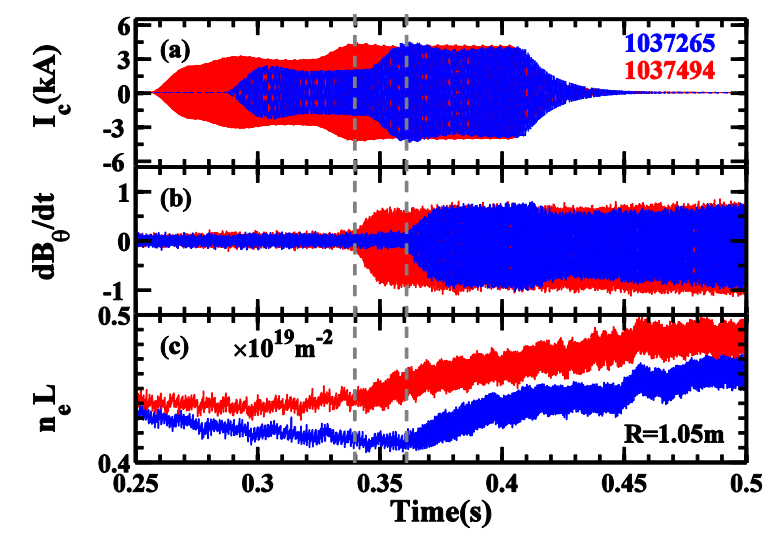

Figure 16. Shot 1037265 and 1037494 , field penetration of rotating 3/1 RMPs. Time evolution of $I_{\mathrm{c}}, \mathrm{d} B_{\theta} / \mathrm{d} t$, and line-integrated density $n_{\mathrm{e}} \mathrm{L}$ at $R=1.05 \mathrm{~m}$.

In order to study the effect of rotating RMPs amplitude on the change of electron density, the waveform of $I_{\mathrm{c}}$ is controlled to ramp up with two flattops as shown in figure 16. For shot 1037265 , $I_{\mathrm{c}}$ is set to ramp up to $2 \mathrm{kA}$ and keeps $50 \mathrm{~ms}$ at the first flattop with field penetration. It is found that the rotating RMPs have no effect on electron density and edge recycling. Here the decrease in $n_{\mathrm{e}} \mathrm{L}$ is mainly due to the decreasing gas puffing fueling. At $t=0.36 \mathrm{~s}$, when $I_{\mathrm{c}}$ further ramps up to $4 \mathrm{kA}$ at the second flattop, field penetration happens and $n_{\mathrm{e}} \mathrm{L}$ begins to increase. For shot $1037494, I_{\mathrm{c}}$ is set to ramp up to $3 \mathrm{kA}$ at the first flattop and $4 \mathrm{kA}$ at the second flattop, and similar results to shot 1037265 are observed when field penetration happens. For both the shots, the global density is increased after field penetration. Additional studies have also been carried out to look into the effect of the rotating frequency of RMPs, it is found that field penetration happens only when $f_{\mathrm{RMP}}$ is high enough $(5.3$ and $6.4 \mathrm{kHz})$ with maximum $I_{\mathrm{c}}$.

\section{Discussion and summary}

By using both static and rotating RMPs with a large 3/1 component, the RMP effect on electron density have been studied on J-TEXT tokamak in the conditions of edge safety factor $q_{a} \sim 3.2-3.3$ with core plasma rotating in the electron diamagnetic drift direction. For tearing-stable plasmas, the detailed time evolution of density profile shows that the $n_{\mathrm{e}} \mathrm{L}$ increases (decreases) inside (around/outside of) the 3/1 RS at first, and the global density is increased later. After field penetration, spontaneous unlocking-penetration circles occur due to the changed density when the RMP amplitude is not strong enough. While for strong enough RMPs, the 2/1 locked mode is also excited, and the global density is further increased. The coil current required for field penetration increases with the electron density, and it is proportionally to the $n_{\mathrm{e}} \mathrm{L}$ at the $3 / 1 \mathrm{RS}$ but to $\left(n_{\mathrm{e}} \mathrm{L}\right)^{0.73}$ for the density at plasma core. The penetration of 3/1 RMPs causes an increase of $\sim 4 \mathrm{~km} / \mathrm{s}$ for $\mathrm{V}_{\phi}(\mathrm{CV})$ in the co- $I_{\mathrm{p}}$ direction, little change in $\mathrm{V}_{\phi}(\mathrm{CIII})$, and an increase of $1 \mathrm{~km} / \mathrm{s}$ and $3 \mathrm{~km} / \mathrm{s}$ for $\mathrm{V}_{\theta}(\mathrm{CV})$ and $\mathrm{V}_{\theta}(\mathrm{CIII})$ towards ion diamagnetic drift direction. In addition, for rotating RMPs with a large 3/1 component, field penetration causes global increase in electron density.

Regarding the effect of RMPs on electron density, the fast decrease of the electron density right after field penetration, as shown in figures 4 and 5, seems to be consistent with the two fluid theory [27], since the plasma rotation is along the electron drift direction. However, the mechanism for the fast increase in the central electron density right after field penetration has not been identified yet. It is possible due to the change of plasma turbulence level in the central region, since plasma rotation is changed by the RMP. The applied RMPs are shown to be able to change the turbulence transport [31,51-54]. The global increase of the electron density well after the field penetration might also be associated the enhanced edge recycling. For rotating RMPs with a large 3/1 component, the increased electron density is consistent with previous finding that a sufficiently high rotating 
frequency of RMPs increases the electron density [33], but the enhanced edge recycling might also play a role here.

Previous nonlinear numerical calculations based two-fluids equations reveal that with increasing the RMP amplitude, the local plasma rotation frequency is forced to approach the local electron diamagnetic drift frequency and to rotate towards the ion drift direction [55], providing a possible explanation for the plasma rotation acceleration by DED observed on TEXTOR [26]. For the experimental results with static 3/1 RMP presented in this paper, the increased electron density results in a stronger density gradient and hence a larger electron diamagnetic drift frequency around the 3/1 RS. According to the results in [55], the plasma will be forced to rotate towards the ion diamagnetic drift direction in order to make the total rotation speed of electron fluid to be zero, being consistent with the experimental results as presented in section 2.3.

In summary, the influence of both static and rotating RMPs on the change of electron density has been studied on J-TEXT Ohmic plasmas. The major results are as follows:

(1) Electron density first increases (decreases) inside (around/outside of) the 3/1 RS right after field penetration, and the global density is increased later associated with increased edge recycling.

(2) For static RMPs, after field penetration either unlocking-penetration circle or excitation of 2/1 locked mode is observed due to the changed density or RMP amplitude.

(3) The penetration threshold of 3/1 RMPs scales linearly to the $n_{\mathrm{e}}$ around the $3 / 1 \mathrm{RS}$ but to $n_{\mathrm{e}}^{0.73}$ for the density at plasma core.

(4) Field penetration of 3/1 RMPs usually causes an increase of $\sim 4 \mathrm{~km} / \mathrm{s}$ for $\mathrm{V}_{\phi}(\mathrm{CV})$ in the co- $I_{\mathrm{p}}$ direction but little change in $\mathrm{V}_{\phi}(\mathrm{CIII})$, and $\mathrm{V}_{\theta}(\mathrm{CV})$ and $\mathrm{V}_{\theta}(\mathrm{CIII})$ is increased for about $1 \mathrm{~km} / \mathrm{s}$ and $3 \mathrm{~km} / \mathrm{s}$ towards the ion diamagnetic drift direction.

\section{Acknowledgement:}

The authors would like to thank the members of the J-TEXT team for their assistance in the experiment. This work is supported by National Magnetic Confinement Fusion Science Program of China (Contract No. 2015GB111001 and 2014GB118000) and the National Natural Science Foundation of China (Contract No.11505069, 11275080, 11305069 and 11405068).

\section{Reference}

[1] Evans T. E. et al 2004 Physical Review Letters 92235003

[2] Evans T. E. et al 2006 nature physics 2419

[3] Liang Y. et al 2007 Physical Review Letters 98265004

[4] Suttrop W. et al 2011 Physical Review Letters 106225004

[5] Jeon Y. M. et al 2012 Physical Review Letters 109035004

[6] Kirk A. et al 2013 Nuclear Fusion 53043007

[7] Jong-Kyu P. et al 2008 Nuclear Fusion 48045006

[8] Buttery R. J. et al 2012 Physics of Plasmas 19056111

[9] Paz-Soldan C. et al 2014 Nuclear Fusion 54073013

[10] Shiraki D. et al 2014 Nuclear Fusion 54033006

[11] Strait E. J. et al 2014 Nuclear Fusion 54073004

[12] Kirk A. et al 2014 Plasma Physics and Controlled Fusion 56104003

[13] In Y. et al 2015 Nuclear Fusion 55043004

[14] Liu Y. Q. et al 2000 Physics of Plasmas 73681

[15] Sabbagh S. A. et al 2006 Physical Review Letters 97045004

[16] Rath N. et al 2013 Nuclear Fusion 53073052

[17] Paz-Soldan C. et al 2015 Nuclear Fusion 55083012 
[18] Ryter F. et al 2012 Nuclear Fusion 52114014

[19] Scannell R. et al 2015 Plasma Physics and Controlled Fusion 57075013

[20] Mordijck S. et al 2015 Plasma Physics and Controlled Fusion 58014003

[21] Schmitz O. et al 2009 Journal of Nuclear Materials 390-91 330

[22] Schmitz O. et al 2012 Nuclear Fusion 52043005

[23] Jakubowski M. W. et al 2013 Nuclear Fusion 53113012

[24] Evans T. E. et al 1992 Journal of Nuclear Materials 196-198 421

[25] Coenen J. W. et al 2011 Nuclear Fusion 51063030

[26] Finken K. H. et al 2007 Physical Review Letters 98065001

[27] Yu Q., Gunter S. 2009 Nuclear Fusion 49062001

[28] Yu Q., Gunter S. 2011 Nuclear Fusion 51073030

[29] Tamain P. et al 2010 Plasma Physics and Controlled Fusion 52075017

[30] Kirk A. et al 2015 Nuclear Fusion 55043011

[31] Mordijck S. et al 2012 Physics of Plasmas 19056503

[32] Hu Q. et al 2014 Nuclear Fusion 54064013

[33] Hu Q. et al 2014 Nuclear Fusion 54122006

[34] Zhuang G. et al 2011 Nuclear Fusion 51094020

[35] Rao B. et al 2014 Fusion Engineering and Design 89378

[36] Chen J. et al 2014 Review of Scientific Instruments 85 11D303

[37] Ding Y. H. et al 2014 Review of Scientific Instruments 85043502

[38] Yang Z. J. et al 2012 Review of Scientific Instruments 83 10E313

[39] Cheng Z. F. et al 2013 Review of Scientific Instruments 84073508

[40] Hender T. C. et al 1992 Nuclear Fusion 322091

[41] Hu Q. et al 2012 Nuclear Fusion 52083011

[42] Hu Q. et al 2013 Physics of Plasmas 20092502

[43] Frassinetti L. et al 2014 Plasma Physics and Controlled Fusion 56104001

[44] Schmitz O. et al 2014 Nuclear Fusion 54012001

[45] Buttery R. J. et al 1999 Nuclear Fusion 391827

[46] Buttery R. J. et al 2000 Nuclear Fusion 40807

[47] Howell D. F. et al 2007 Nuclear Fusion 471336

[48] Loewenbrueck K. et al 2007 34th EPS Conference on Plasma Phys (Warsaw, Poland, 2007) vol 31F (ECA), p -1.136 http://epsppd.epfl.ch/Warsaw/pdf/P1_136.pdf

[49] Wang N. et al 2014 Nuclear Fusion 54064014

[50] Jin H. et al 2015 Plasma Physics and Controlled Fusion 57104007

[51] Schmitz O. et al 2008 Nuclear Fusion 48024009

[52] Xu Y. et al 2006 Physical Review Letters 97165003

[53] Xu Y. et al 2007 Nuclear Fusion 471696

[54] Mordijck S. et al 2012 Physics of Plasmas 19024504

[55] Yu Q., et al 2009 Physics of Plasmas 16042301 